\title{
Neural Network Control of a Robot Interacting With an Uncertain Viscoelastic Environment
}

\author{
S. Bhasin, K. Dupree, P. M. Patre, and W. E. Dixon
}

\begin{abstract}
A continuous controller is developed for a robot that moves in free space, undergoes a collision with a viscoelastic environment, and then regulates the new coupled dynamic system to a desired setpoint. Since the model for the viscoelastic surface contains uncertainties that do not satisfy the linear-in-the-parameters assumption, the model is approximated by a neural network feedforward term, which is combined with a continuous feedback term to guarantee uniformly ultimately bounded regulation of the system despite parametric uncertainties in the robot and the viscoelastic environment. Experimental results of a two-link robot interacting with a human tissue phantom demonstrate the performance of the proposed controller.
\end{abstract}

Index Terms-Backstepping, contact transition control, human-robot interaction, Lyapunov methods, nonlinear control, robot control, soft/viscoelastic impact.

\section{INTRODUCTION}

$\mathbf{R}$ OBOTIC systems are often required to physically interact with an environment; yet, this interaction raises stability and performance concerns. One strategy to account for potentially destabilizing impact forces is to model the robot-environment interaction and include these dynamics in the control design and stability analysis. Hence, significant efforts have focused on developing robot-environment contact models.

Hertz [1] developed one of the first contact models, where a linear spring is used to relate the impact force to local deformation. Since Hertz's early work, different contact models have been developed that can also account for energy dissipation effects at impact through the inclusion of a damping term. The models range from the simple linear Kelvin-Voigt model to the more complex impact pair model [2]. Hunt and Crossley [3] proposed a nonlinear compliant contact model, which not only included both stiffness and damping terms, but also eliminated the discontinuous impact forces at initial contact and separation, thus making it more suitable for robotic contact with compliant environments. Marhefka and Orin [4] used the Hunt-Crossley model for simulation of robotic systems undergoing a contact transition, and more recently, the model was used in an online

Manuscript received November 23, 2009; revised April 07, 2010; accepted June 18, 2010. Manuscript received in final form June 18, 2010. Date of publication July 19, 2010; date of current version June 17, 2011. Recommended by Associate Editor A. Loria. This work was supported in part by the NSF CAREER Award 0547448, NSF Award 0901491, and by the Department of Energy under Grant DE-FG04-86NE37967 as part of the DOE University Research Program in Robotics (URPR).

S. Bhasin, K. Dupree, P. M. Patre, and W. E. Dixon are with the Department of Mechanical and Aerospace Engineering, University of Florida, Gainesville FL 32611-6250 USA (e-mail: sbhasin@ufl.edu; kdupree@ufl.edu; pmpatre@ufl.edu; wdixon@ufl.edu).

Color versions of one or more of the figures in this brief are available online at http://ieeexplore.ieee.org.

Digital Object Identifier 10.1109/TCST.2010.2054094 estimation algorithm to estimate the mechanical impedance parameters during contact [5]. Because the model has been shown to better represent the physical nature of the energy transfer process at contact [6], it has found acceptance in the scientific community [4]-[6].

Given some model of the impact dynamics, another challenge is to develop a closed loop controller for a robot, as it transitions from a non-contact to a contact state. The destabilizing impact forces and the uncertainty in the environment dynamics make it a complex control problem. A variety of techniques have been developed to control the robot motion in the presence of a contact transition [7]-[19]. Hogan [7] developed an impedance control technique for contact transition with a stiff environment. A hybrid method for control of position and force was proposed in [8]. Yousef-Toumi and Gutz [9] used integral force compensation with velocity feedback to improve transient impact response. Mills and Lokhorst [10] proved stability of contact transition using a discontinuous controller for compliant environments. Tarn et al. [11] used positive acceleration feedback together with a switching control strategy for force regulation and contact transition control. Discontinuous control approaches are also proposed in [12], [13]. Stability of a system undergoing contact transition is investigated using hybrid systems theory in [19] and [20]. In [20], the robot is modeled as a switched system, which uses a proportional-differential (PD) controller for position control in the non-contact phase and a parallel force/position controller in the contact phase. Recently, intelligent control approaches have been explored for coordinated control of mobile manipulators in contact with a compliant environment [21]. Some of the limitations of the control approaches discussed above are the use of a simplistic contact model, and the need for exact knowledge of the robot and/or environment dynamics.

The development in this brief and our previous work in [18] and [22] investigate the problem of robot contact transition with a viscoelastic environment. In our previous efforts [16], [23], a linear spring contact model was used to develop a controller for robot contact transition with a stiff environment. The contribution of the work was the development of a single continuous controller for both the non-contact and contact states of the robot. Experiments were performed where the robot collided with a stiff aluminum surface. Motivated by applications where the robot will collide with a compliant/viscoelastic surface, our preliminary work in [17] used the more general Hunt-Crossley contact model to account for the energy dissipation at contact. However, the development in [17] assumed knowledge of the local deformation of the material raised to the Hertzian compliance exponent, which is usually difficult to determine [24]. One reason that exact knowledge of the Hertzian compliance exponent is required in [17] is that this effect does not satisfy 
the linear-in-the-parameters (LP) assumption. This limitation is overcome in this brief by the use of neural networks (NNs), which can approximate non-LP dynamics. Uniformly ultimately bounded (UUB) stability of the controller is proven using a Lyapunov-based stability analysis. Experiments with a human tissue phantom illustrate the performance of the controller.

In comparison to previous results in literature, efforts in this brief are motivated by the desire to develop a continuous controller that can guide a robot from a non-contact position to contact with an uncertain environment and then regulate the coupled dynamic system to a desired setpoint. Also, many regulation problems in robot control literature, constrained or unconstrained, can be solved using a model-free approach. A simple proportional-integral-differential (PID) controller can be used to regulate the robot end effector without the need to know or adapt for the robot dynamics. However, the problem scenario considered in this brief is fundamentally different. The constrained space of the robot is not static rather it is a dynamic system (viscoelastic mass-spring-damper system, also referred to as the environment) and the objective is to regulate the state of the dynamic environment and not the robot end-effector, which is the case with most regulation results in literature. The uncertain viscoelastic effects at the contact interface between the robot and the dynamic environment are accounted for by including a physically consistent contact model in the closed loop error dynamics. A backstepping approach is used to control the robot states such that the environment position is driven to a desired set-point. NNs are used to approximate the unknown dynamics of the environment and the robot, and should normally lead to a model-free approach; however, to satisfy the continuity requirement of the controller certain discontinuous, non-differentiable terms are left out of the NN approximation. These model based terms are then treated as a disturbance and handled using continuous robust control methods.

\section{DYNAMIC MODEL}

The subsequent development is motivated by the scenario in which a robot is controlled to move from a non-contact state to a contact state with a viscoelastic environment such that the environment is regulated to a desired position. The viscoelastic environment is modeled by a mass-spring-damper system with a viscoelastic contact interface The dynamic model for a rigid two-link revolute robot in contact with a compliant viscoelastic environment is given by

$$
\begin{aligned}
M\left(x_{r}\right) \ddot{x}_{r}+C\left(x_{r}, \dot{x}_{r}\right) \dot{x}_{r}+h\left(x_{r}\right)+\left[\begin{array}{c}
F_{m} \\
0
\end{array}\right] & =F \\
m \ddot{x}_{m}+b_{m} \dot{x}_{m}+k_{s}\left(x_{m}-x_{0}\right) & =F_{m} .
\end{aligned}
$$

In (1), $x_{r}(t), \dot{x}_{r}(t), \ddot{x}_{r}(t) \in \mathbb{R}^{2}$ represent the planar Cartesian position, velocity, and acceleration of the robot end-effector, respectively, $M\left(x_{r}\right) \in \mathbb{R}^{2 \times 2}$ represents the uncertain inertia matrix, $C\left(x_{r}, \dot{x}_{r}\right) \in \mathbb{R}^{2 \times 2}$ represents the uncertain centripetal-Coriolis effects, $h\left(x_{r}\right) \in \mathbb{R}^{2}$ represents uncertain conservative forces (e.g., gravity), $F_{m}(t) \in \mathbb{R}$ denotes the interaction force between the robot and the environment during impact, and $F(t) \in \mathbb{R}^{2}$ represents the force control inputs. In (2), $x_{m}(t), \dot{x}_{m}(t), \ddot{x}_{m}(t) \in \mathbb{R}$ represent the displacement, velocity, and acceleration of the unknown mass $m \in \mathbb{R}, x_{0} \in \mathbb{R}$ represents the initial undisturbed position of the mass, $b_{m} \in \mathbb{R}$ is the damping constant, and $k_{s} \in \mathbb{R}$ represents the unknown stiffness of the spring connected to the mass. When the horizontal position of the robot, denoted by $x_{r 1}(t) \in \mathbb{R}$, is greater than or equal to the position of the viscoelastic material (i.e., when $\left.x_{r 1}(t) \geq x_{m}(t)\right)$ contact occurs, and the interaction force $F_{m}(t)$ is modeled using the Hunt-Crossley contact model as [22]

$$
F_{m} \triangleq \lambda \delta^{n}+b \dot{\delta} \delta^{n}
$$

where $\lambda \in \mathbb{R}$ is the unknown stiffness of the contact interface, $b \in \mathbb{R}$ is the unknown impact damping coefficient, and $\delta(t) \in \mathbb{R}$ denotes the local deformation of the material defined as

$$
\delta \triangleq \begin{cases}0, & x_{r 1}<x_{m} \\ x_{r 1}-x_{m}, & x_{r 1} \geq x_{m}\end{cases}
$$

Also in (3), $\dot{\delta}(t)$ is the relative velocity of the contacting bodies, and $n \in \mathbb{R}$ is the unknown Hertzian compliance coefficient which depends on the surface geometry of the contact. The model in (3) is a continuous contact force-based model wherein the contact forces increase from zero upon impact and return to zero upon separation. Also, the energy dissipation during impact is a function of the damping constant which can be related to the impact velocity and the coefficient of restitution [3]. The contact is considered to be direct-central and quasi-static (i.e., all the stresses are transmitted at the time of contact and sliding and friction effects during contact are negligible) where plastic deformation effects are assumed to be negligible. The following skew-symmetric relationship is satisfied [25]

$$
\xi^{T}\left(\frac{1}{2} \dot{M}\left(x_{r}\right)-C\left(x_{r}, \dot{x}_{r}\right)\right) \xi=0 \quad \forall \xi \in \mathbb{R}^{2} .
$$

Assumption 1: The inertia matrix $M\left(x_{r}\right)$ is symmetric, positive definite, and can be lower and upper bounded as

$$
a_{1}\|\xi\|^{2} \leq \xi^{T} M \xi \leq a_{2}\|\xi\|^{2} \quad \forall \xi \in \mathbb{R}^{2}
$$

where $a_{1}, a_{2} \in \mathbb{R}$ are positive constants and $\|\cdot\|$ denotes the standard Euclidean norm. Also, the minimum singular value of the Jacobian, $J(q)$ is greater than a known, small positive constant $\epsilon>0$, such that $\max \left\{\left\|J^{-1}(q)\right\|\right\}$ is known a priori, and hence, all kinematic singularities are always avoided. Because the Jacobian singularity is avoided the task space inertia matrix $M\left(x_{r}\right)$ can be assumed to be positive definite.

Assumption 2: The robot and mass-spring-damper positions, $x_{r}(t)$ and $x_{m}(t)$, and the corresponding velocities, $\dot{x}_{r}(t)$ and $\dot{x}_{m}(t)$, are measurable. Further, it is assumed that $x_{r}(t)$ is bounded based on the geometry of the robot.

Assumption 3: The local deformation of the viscoelastic material during contact, $\delta(t)$, defined in (4), is assumed to be bounded. Also, the unknown constants in the dynamic models (2) and (3), i.e., $m, b_{m}, k_{s}, \lambda, b, n$ are assumed to be bounded.

Remark 1: While the subsequent control design and stability analysis are developed for a two degree-of-freedom (DOF) system in a planar Cartesian-space, the underlying mathematics can be extended to a higher DOF robot if the environment is constrained to move along a straight line. However, if there are no constraints on the environment, additional development 
would be required to control the position of the environment since the interacting force $F_{m}(t)$, modeled as (3), which is used to control the position of the environment through the robot, acts only along a straight line perpendicular to the surface of the environment.

\section{CONTROl Development}

In the subsequent control development, the desired robot velocity is designed as a virtual control input to the unactuated viscoelastic environment. The desired velocity is designed to ensure that the robot impacts and then regulates the mass to a desired position. Since it is not possible to directly control the mass trajectory, backstepping methods are used to develop a control input to ensure that the robot tracks the desired trajectory despite the non-contact to contact transition and parametric uncertainties in the robot and the environment. The viscoelastic model requires that the backstepping error be developed in terms of the desired robot velocity. A challenge to backstep on the desired robot velocity is that it is premultiplied by $\delta(t)$, which is zero when the robot and mass are not in contact. Hence, a strategic combination of nonlinear damping and $\mathrm{NN}$ backstepping is used in the subsequent development.

\section{A. Control Objective}

The control objective is to regulate the position of the environment via a robot that transitions from non-contact to contact state with the environment through an impact collision. To quantify the control objective, the following errors are defined

$$
e_{r} \triangleq x_{r d}-x_{r}, \quad e_{m} \triangleq x_{m d}-x_{m}
$$

where $e_{r}(t) \triangleq\left[e_{r 1}(t), e_{r 2}(t)\right]^{T} \in \mathbb{R}^{2}$, and $e_{m}(t) \in \mathbb{R}$ denote the errors for the end-effector of the robot and mass-spring-damper system, respectively. In (7), $x_{m d} \in \mathbb{R}$ denotes the constant known desired position of the mass, and $x_{r d}(t) \triangleq\left[x_{r d 1}(t), x_{r d 2}\right]^{T} \in \mathbb{R}^{2}$ denotes the desired position of the end-effector of the robot. To facilitate the subsequent control design and stability analysis, filtered tracking errors for the robot and the mass-spring-damper, denoted by $r_{r}(t) \in \mathbb{R}^{2}$ and $r_{m}(t) \in \mathbb{R}$ respectively, are defined as

$$
r_{r} \triangleq \dot{e}_{r}+\alpha e_{r}, \quad r_{m} \triangleq \dot{e}_{m}+\gamma e_{m}
$$

where $\alpha, \gamma \in \mathbb{R}$ are positive constant gains.

\section{B. NN Feedforward Estimation}

NN-based estimation methods are well suited for control systems where the dynamic model contains uncertainties as in (1)-(3). Multilayer NNs have been shown to be capable of approximating generic nonlinear continuous functions. Let $\mathbb{S}$ be a compact simply connected set of $\mathbb{R}^{N_{1}+1}$. With map $f: \mathbb{S} \rightarrow \mathbb{R}^{n}$, define $\mathbb{C}^{n}(\mathbb{S})$ as the space where $f$ is continuous. There exist weights and thresholds such that some function $f(x) \in \mathbb{C}^{n}(\mathbb{S})$ can be represented by a three-layer $\mathrm{NN}$ as [26]

$$
f(x)=W^{T} \sigma\left(V^{T} x\right)+\varepsilon(x)
$$

for some given input $x(t) \in \mathbb{R}^{N_{1}+1}$. In (9), $V \in \mathbb{R}^{\left(N_{1}+1\right) \times N_{2}}$ and $W \in \mathbb{R}^{\left(N_{2}+1\right) \times n}$ are bounded constant ideal weight matrices for the first-to-second and second-to-third layers, respectively, where $N_{1}$ is the number of inputs in the input layer, $N_{2}$ is the number of neurons in the hidden layer, and $n$ is the number of outputs in the output layer. The activation function in (9) is denoted by $\sigma(\cdot) \in \mathbb{R}^{N_{2}+1}$, and $\varepsilon(x) \in \mathbb{R}^{n}$ is the functional reconstruction error. Based on (9), the typical three-layer NN approximation for $f(x)$ is given as [26]

$$
\hat{f}(x) \triangleq \hat{W}^{T} \sigma\left(\hat{V}^{T} x\right)
$$

where $\hat{V}(t) \in \mathbb{R}^{\left(N_{1}+1\right) \times N_{2}}$ and $\hat{W}(t) \in \mathbb{R}^{\left(N_{2}+1\right) \times n}$ are subsequently designed estimates of the ideal weight matrices. The estimate mismatch for the ideal weight matrices, denoted by $\tilde{V}(t) \in \mathbb{R}^{\left(N_{1}+1\right) \times N_{2}}$ and $\tilde{W}(t) \in \mathbb{R}^{\left(N_{2}+1\right) \times n}$, are defined as $\tilde{V} \triangleq V-\hat{V}$ and $\tilde{W} \triangleq W-\hat{W}$, respectively, and the mismatch for the hidden-layer output error for a given $x(t)$, denoted by $\tilde{\sigma}(x) \in \mathbb{R}^{N_{2}+1}$, is defined as $\tilde{\sigma} \triangleq \sigma-\hat{\sigma}=\sigma\left(V^{T} x\right)-\sigma\left(\hat{V}^{T} x\right)$. The NN has several properties that facilitate the subsequent development.

1) Property 1: The Taylor series expansion for $\sigma\left(V^{T} x\right)$ for a given $x$ may be written as [26]

$$
\sigma\left(V^{T} x\right)=\sigma\left(\hat{V}^{T} x\right)+\sigma^{\prime}\left(\hat{V}^{T} x\right) \tilde{V}^{T} x+O\left(\tilde{V}^{T} x\right)^{2}
$$

where $\sigma^{\prime}\left(\hat{V}^{T} x\right) \equiv d \sigma\left(V^{T} x\right) /\left.d\left(V^{T} x\right)\right|_{V^{T} x=\hat{V}^{T} x}$, and $O\left(\hat{V}^{T} x\right)^{2}$ denotes the higher order terms. Simplifying (11) yields

$$
\tilde{\sigma}=\hat{\sigma}^{\prime} \tilde{V}^{T} x+O\left(\tilde{V}^{T} x\right)^{2}
$$

where $\hat{\sigma}^{\prime} \triangleq \sigma^{\prime}\left(\hat{V}^{T} x\right)$.

2) Property 2: The ideal weights are assumed to exist and be bounded by known positive values, i.e., $\|V\|_{F}^{2} \leq \bar{V}_{B}$ and $\|W\|_{F}^{2} \leq \bar{W}_{B}$, where $\|\cdot\|_{F}$ is the Frobenius norm of a matrix.

3) Property 3: The estimates for NN weights, $\hat{W}(t)$ and $\hat{V}(t)$, can be bounded using the projection algorithm as in [27] and [28].

4) Property 4: The activation function $\sigma(\cdot)$ and its derivative are bounded, e.g., sigmoidal functions, hyperbolic functions, etc.

5) Property 5: On a given compact set $S$, the functional reconstruction error $\varepsilon(x)$ is bounded, i.e., $\|\varepsilon(x)\| \leq \varepsilon_{n}$, where $\varepsilon_{n}$ is a known positive constant.

\section{Closed-Loop Error System}

The open-loop error system for the mass can be obtained by multiplying (8) by $m$ and then taking its time derivative as

$$
m \dot{r}_{m}=f_{1}-\lambda \delta^{n}-b \dot{\delta} \delta^{n}-e_{m}
$$

where the function $f_{1}(t) \in \mathbb{R}$ is defined as

$$
f_{1} \triangleq b_{m} \dot{x}_{m}+k_{s}\left(x_{m}-x_{0}\right)+m \gamma \dot{e}_{m}+e_{m} .
$$

The auxillary function in (14) can be represented by as

$$
f_{1}=W_{1}^{T} \sigma_{1}\left(V_{1}^{T} x_{1}\right)+\varepsilon_{m}\left(x_{1}\right)
$$


where the NN input $x_{1}(t) \in \mathbb{R}^{3}$ is defined as $x_{1} \triangleq\left[1, e_{m}, r_{m}\right]^{T}$, $W_{1} \in \mathbb{R}^{\left(N_{m 2}+1\right) \times 1}$, and $V_{1} \in \mathbb{R}^{3 \times N_{m 2}}$ are ideal NN weights, $N_{m 2} \in \mathbb{R}$ denotes the number of hidden layer neurons, and $\varepsilon_{m} \in \mathbb{R}$ is the functional reconstruction error. Since the openloop error expression for the mass in (13) does not have an actual control input, a virtual control input, $\dot{x}_{r d 1}(t)$, is introduced by adding and subtracting $\left(1-b \delta^{n}\right) \dot{x}_{r d 1}$ to (13) as

$$
\begin{aligned}
m \dot{r}_{m}= & W_{1}^{T} \sigma_{1}\left(V_{1}^{T} x_{1}\right)+\varepsilon_{m}\left(x_{1}\right)-\lambda \delta^{n}-b \dot{\delta} \delta^{n} \\
& -e_{m}+\left(1-b \delta^{n}\right) \dot{x}_{r d 1}-\left(1-b \delta^{n}\right) \dot{x}_{r d 1} .
\end{aligned}
$$

To facilitate the subsequent backstepping-based design, the virtual control input to the unactuated mass-spring-damper system is designed as

$$
\dot{x}_{r d 1}=\hat{W}_{1}^{T} \sigma_{1}\left(\hat{V}_{1}^{T} x_{1}\right)-k_{m} r_{m} .
$$

In (17), $k_{m} \in \mathbb{R}$ is a constant positive control gain, and $\hat{W}_{1}(t) \in$ $\mathbb{R}^{\left(N_{m 2}+1\right) \times 1}$ and $\hat{V}_{1}(t) \in \mathbb{R}^{3 \times N_{m 2}}$ are the estimates of the ideal weights, which are updated based on the subsequent stability analysis as

$$
\begin{aligned}
& \dot{\hat{W}}_{1}=\operatorname{proj}\left(\Gamma_{w 1} \hat{\sigma}_{1} r_{m}-\Gamma_{w 1} \hat{\sigma}_{1}^{\prime} \hat{V}_{1}^{T} x_{1} r_{m}\right) \\
& \dot{\hat{V}}_{1}=\operatorname{proj}\left(\Gamma_{v 1} x_{1} \hat{W}_{1}^{T} \hat{\sigma}_{1}^{\prime} r_{m}\right)
\end{aligned}
$$

where $\Gamma_{w 1} \in \mathbb{R}^{\left(N_{m 2}+1\right) \times\left(N_{m 2}+1\right)}, \Gamma_{v 1} \in \mathbb{R}^{3 \times 3}$ are constant, positive definite, symmetric gain matrices, and $\operatorname{proj}(\cdot)$ denotes a projection algorithm ${ }^{1}$ utilized to guarantee that the weight estimates $\hat{W}_{1}(t)$ and $\hat{V}_{1}(t)$ remain bounded [27], [28]. The estimates for the NN weights in (18) are generated online (there is no offline learning phase). Also, $x_{r d 2}=\rho$, where $\rho \in \mathbb{R}$ is an appropriate positive constant, selected so the robot will impact the mass-spring-damper system. $x_{r d 2}(t)$ is the desired robot position measured tangential to the tissue surface. Since the mass is to be controlled in the direction normal to the tissue surface, it is only required that $x_{r d 2}(t)$ be chosen such that the robot impacts the tissue surface. The closed-loop error system for the mass can be developed by substituting (17) into (16) as

$$
\begin{aligned}
m \dot{r}_{m}= & W_{1}^{T} \sigma_{1}\left(V_{1}^{T} x_{1}\right)-\hat{W}_{1}^{T} \sigma_{1}\left(\hat{V}_{1}^{T} x_{1}\right)+\varepsilon_{m}\left(x_{1}\right) \\
& -k_{m} r_{m}-\lambda \delta^{n}+b \delta^{n} \dot{e}_{r 1}-b \delta^{n} \dot{e}_{m} \\
& -e_{m}+\left(1-b \delta^{n}\right) \dot{x}_{r d 1} .
\end{aligned}
$$

Adding and subtracting $W_{1}^{T} \hat{\sigma}_{1}+\hat{W}_{1}^{T} \tilde{\sigma}_{1}$ to (19), and then using the Taylor series approximation in (12), the following expression for the closed-loop mass error system can be obtained

$m \dot{r}_{m}=\tilde{W}_{1}^{T} \hat{\sigma}_{1}-\tilde{W}_{1}^{T} \hat{\sigma}_{1}^{\prime} \hat{V}_{1}^{T} x_{1}+\hat{W}_{1}^{T} \hat{\sigma}_{1}^{\prime} \tilde{V}_{1}^{T} x_{1}-k_{m} r_{m}-e_{m}+w_{1}$

where the notation $\hat{\sigma}_{1}$ and $\tilde{\sigma}_{1}$ is introduced in (12), and $w_{1}(t) \in$ $\mathbb{R}$ is defined as

$$
w_{1}=\tilde{W}_{1}^{T} \hat{\sigma}_{1}^{\prime} V_{1}^{T} x_{1}+W_{1}^{T} O\left(\tilde{V}_{1}^{T} x_{1}\right)^{2}+\varepsilon_{m}\left(x_{1}\right)-\lambda \delta^{n}
$$

${ }^{1}$ The initial weights are selected in some compact set, then new estimates are generated based on the weight update laws in (18). If the weights on the boundary of the set are found to be directed outside the compact set, they are projected back into the set using a smooth projection algorithm (see [27], [28] for details).

$$
+b \delta^{n} \dot{e}_{r 1}-b \delta^{n} \dot{e}_{m}+\left(1-b \delta^{n}\right) \dot{x}_{r d 1} .
$$

Using Property 2-5 and [26], $w_{1}(t)$ can be bounded ${ }^{2}$ as

$$
\left|w_{1}\right| \leq c_{m 1}+c_{m 2}\|z\|
$$

where $c_{m 1}, c_{m 2} \in \mathbb{R}$ are computable known positive constants and $z \in \mathbb{R}^{6}$ is defined as

$$
z \triangleq\left[\begin{array}{llll}
e_{m} & r_{m} & e_{r}^{T} & r_{r}^{T}
\end{array}\right]
$$

The open-loop robot error system can be obtained by taking the time derivative of $r_{r}(t)$, premultiplying by the robot inertia matrix $M\left(x_{r}\right)$, and utilizing (1), (7), and (8) as

$$
M \dot{r}_{r}=f_{2}-C r_{r}-F
$$

where the function $f_{2}(t) \in \mathbb{R}^{2}$ is defined as

$f_{2} \triangleq M \ddot{x}_{r d}+M \alpha \dot{e}_{r}+h+C \dot{x}_{r d}+C \alpha e_{r}+\left[\begin{array}{c}\lambda \delta^{n}+b \dot{\delta} \delta^{n} \\ 0\end{array}\right]$.

By representing the function $f_{2}(t)$ by a $\mathrm{NN}$, the expression in (24) can be written as

$$
M \dot{r}_{r}=W_{2}^{T} \sigma_{2}\left(V_{2}^{T} x_{2}\right)+\varepsilon_{r}\left(x_{2}\right)-C r_{r}-F
$$

where the NN input $x_{2}(t) \in \mathbb{R}^{13}$ is defined as $x_{2}(t) \triangleq$ $\left[1, \delta, x_{r}^{T}, x_{r d}^{T}, \delta \dot{\delta}, \dot{x}_{r}^{T}, \dot{x}_{r d}^{T}, \ddot{x}_{r d}^{T}\right]^{T}, W_{2} \in \mathbb{R}^{\left(N_{r 2}+1\right) \times 2}$, and $V_{2} \in \mathbb{R}^{13 \times N_{r 2}}$ are ideal NN weights, $N_{r 2} \in \mathbb{R}$ denotes the number of hidden layer neurons, $\varepsilon_{r} \in \mathbb{R}$ is the functional reconstruction error. From the choice of the virtual control input in (17), an expression for $\ddot{x}_{r d 1}(t)$ can be developed which is continuous and does not require acceleration measurements. This fact can be used to show that the input $x_{2}(t)$ to the NN is continuous and known. Based on (26) and the subsequent stability analysis, the robot force control input is designed as

$$
F=\hat{W}_{2}^{T} \sigma_{2}\left(\hat{V}_{2}^{T} x_{2}\right)+k_{r} r_{r}+e_{r}
$$

where $k_{r} \in \mathbb{R}$ is a constant positive control gain, and $\hat{W}_{2}(t) \in$ $\mathbb{R}^{\left(N_{r 2}+1\right) \times 2}$ and $\hat{V}_{2}(t) \in \mathbb{R}^{13 \times N_{r 2}}$ are the estimates of the ideal weights, which are designed based on the subsequent stability analysis as

$$
\begin{aligned}
& \dot{\hat{W}}_{2}=\operatorname{proj}\left(\Gamma_{w 2} \hat{\sigma}_{2} r_{r}^{T}-\Gamma_{w 2} \hat{\sigma}_{2}^{\prime} \hat{V}_{2}^{T} x_{2} r_{r}^{T}\right) \\
& \dot{\hat{V}_{2}}=\operatorname{proj}\left(\Gamma_{v 2} x_{2} r_{r}^{T} \hat{W}_{2}^{T} \hat{\sigma}_{2}^{\prime}\right)
\end{aligned}
$$

where $\Gamma_{w 2} \in \mathbb{R}^{\left(N_{r 2}+1\right) \times\left(N_{r 2}+1\right)}, \Gamma_{v 2} \in \mathbb{R}^{13 \times 13}$ are constant, positive definite, symmetric gain matrices. Substituting (27) in (26) and following a similar approach as in the mass error system in (19)-(20), the closed-loop error system for the robot is obtained as

$$
\begin{aligned}
M \dot{r}_{r}= & \tilde{W}_{2}^{T} \hat{\sigma}_{2}-\tilde{W}_{2}^{T} \hat{\sigma}_{2}^{\prime} \hat{V}_{2}^{T} x_{2}+\hat{W}_{2}^{T} \hat{\sigma}_{2}^{\prime} \tilde{V}_{2}^{T} x_{2} \\
& -C r_{r}-k_{r} r_{r}-e_{r}+w_{2}
\end{aligned}
$$

${ }^{2}$ Since the term $w_{1}(t)$ in (21) consists of approximation errors and terms which cannot be adapted for, it is treated as disturbance and handled using robust control techniques. Also, $w_{1}(t)$ contains the estimation error $\tilde{W}_{1}(t)$ which can be shown to be bounded, i.e., $\tilde{W}_{1} \in \mathcal{L}_{\infty}$, since $\hat{W}_{1}$ in (18) is bounded by projection and $W_{1}$ is a constant ideal weight matrix. 

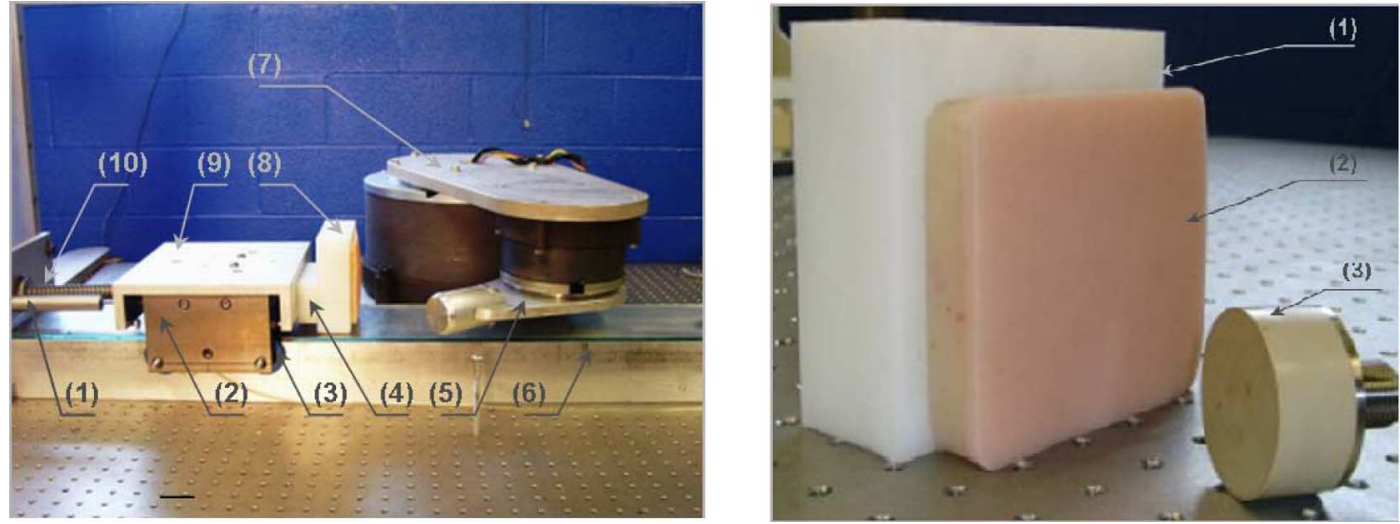

Fig. 1. Top view of the experimental testbed including: (1) LVDT, (2) undercarriage, (3) air bearings, (4) sensor housing, (5) Link 2, (6) aluminum rail, (7) Link 1, (8) tissue housing, (9) U-shaped aluminum plate, (10) spring. (Right) (1) Tissue phantom housing, (2) viscoelastic tissue phantom, (3) inductive sensor.

where $w_{2}(t) \in \mathbb{R}^{2}$ is a disturbance term defined as

$$
w_{2}=\tilde{W}_{2}^{T} \hat{\sigma}_{2}^{\prime} V_{2}^{T} x_{2}+W_{2}^{T} O\left(\tilde{V}_{2}^{T} x_{2}\right)^{2}+\varepsilon_{r}\left(x_{2}\right) .
$$

Using Property $2-5$ and [26], $w_{2}(t)$ can be bounded as

$$
\left\|w_{2}\right\| \leq c_{r 1}+c_{r 2}\|z\|
$$

where $c_{r 1}, c_{r 2} \in \mathbb{R}$ are computable known positive constants.

\section{STABILITy ANALYSIS}

Theorem: The controller given by (17), (18), (27), and (28) ensures uniformly ultimately bounded regulation of the states of the robot and mass-spring-damper system as

$$
\|z(t)\| \leq \varepsilon_{1} e^{-\varepsilon_{2} t}+\varepsilon_{0}
$$

provided the control gains are selected according to the sufficient gain conditions

$$
\min \left(\gamma, 1, \lambda_{\min }\{\alpha\}\right)>\frac{c_{m 2}^{2}}{4 k_{m}}+\frac{c_{r 2}^{2}}{4 k_{r}}
$$

where $\varepsilon_{0}, \varepsilon_{1}, \varepsilon_{2} \in \mathbb{R}$ denote positive constants; $\lambda_{\min }\{\cdot\}$ denotes the minimum Eigenvalue of a matrix, the control gains $k_{m}$ and $k_{r}$ are introduced in (17) and (27), respectively; $c_{m 2}$ and $c_{r 2}$ are introduced in (22) and (31).

Proof: Let $V(t) \in \mathbb{R}$ denote a positive definite, radially unbounded function defined as

$$
\begin{aligned}
V= & \frac{1}{2} r_{r}^{T} M r_{r}+\frac{1}{2} e_{r}^{T} e_{r}+\frac{1}{2} \operatorname{tr}\left(\tilde{W}_{1}^{T} \Gamma_{w 1}^{-1} \tilde{W}_{1}\right) \\
& +\frac{1}{2} \operatorname{tr}\left(\tilde{V}_{1}^{T} \Gamma_{v 1}^{-1} \tilde{V}_{1}\right)+\frac{1}{2} \operatorname{tr}\left(\tilde{W}_{2}^{T} \Gamma_{w 2}^{-1} \tilde{W}_{2}\right) \\
& +\frac{1}{2} \operatorname{tr}\left(\tilde{V}_{2}^{T} \Gamma_{v 2}^{-1} \tilde{V}_{2}\right)+\frac{1}{2} m r_{m}^{2}+\frac{1}{2} e_{m}^{2} .
\end{aligned}
$$

Using (5), Assumption 3, (8), (18), (20), (22), (28), (29), and (31), an upper bound for time derivative of $V(t)$ can be determined as

$$
\begin{aligned}
\dot{V} \leq & -k_{r}\left\|r_{r}\right\|^{2}-\lambda_{\min }\{\alpha\}\left\|e_{r}\right\|^{2}-k_{m} r_{m}^{2}-\gamma e_{m}^{2} \\
& +\left(c_{m 1}+c_{m 2}\|z\|\right)\left|r_{m}\right|+\left(c_{r 1}+c_{r 2}\|z\|\right)\left\|r_{r}\right\| .
\end{aligned}
$$

The control gains $k_{m}$ and $k_{r}$ are now defined as

$$
k_{m} \triangleq k_{m 1}+k_{m 2}+1 ; \quad k_{r} \triangleq k_{r 1}+k_{r 2}+1
$$

where $k_{m i}, k_{r i} \in \mathbb{R},(i=1,2)$ are positive constant nonlinear damping gains. Substituting for $k_{m}$ and $k_{r}$, the expression in (35) can be rewritten as

$$
\begin{aligned}
\dot{V} \leq & -\gamma e_{m}^{2}-r_{m}^{2}-\lambda_{\min }\{\alpha\}\left\|e_{r}\right\|^{2}-\left\|r_{r}\right\|^{2} \\
& -\left[k_{m 1} r_{m}^{2}-c_{m 1}\left|r_{m}\right|\right]-\left[k_{m 2} r_{m}^{2}-c_{m 2}\|z\|\left|r_{m}\right|\right] \\
& -\left[k_{r 1}\left\|r_{r}\right\|^{2}-c_{r 1}\left\|r_{r}\right\|\right]-\left[k_{r 2}\left\|r_{r}\right\|^{2}-c_{r 2}\|z\|\left\|r_{r}\right\|\right] .
\end{aligned}
$$

Completing the squares on the bracketed terms and provided the sufficient gain condition in (33) is satisfied, the expression in (37) can be reduced to

$$
\dot{V} \leq-\beta\|z\|^{2}+\frac{c_{m 1}^{2}}{4 k_{m 1}}+\frac{c_{r 1}^{2}}{4 k_{r 1}}
$$

where $\beta \in \mathbb{R}$ is a positive constant. The expression in (38) can be used to show that $\dot{V}(t)$ is negative whenever $z(t)$ lies outside the compact set $\Omega_{z} \triangleq\left\{z:\|z\| \leq \sqrt{c_{m 1}^{2} / 4 k_{m 1}+c_{r 1}^{2} / 4 k_{r 1} / \beta}\right\}$, and hence, $\|z(t)\|$ is UUB [29]. The size of $\Omega_{z}$ can be made arbitrarily small by increasing the control gains $k_{m}$ and $k_{r}$. Using the definition of $z(t)$ in (23), $r_{r}(t), e_{r}(t), r_{m}(t), e_{m}(t) \in \mathcal{L}_{\infty}$, and hence, $F(t)$ and all closed-loop signals remain bounded. Standard signal chasing analysis can be used to show that all signals remain bounded.

Remark 2: The above analysis assumes that the NN approximation property holds throughout. However, according to Property 7 , the NN universal approximation property only holds on a compact set. To show that the NN inputs always stay inside a compact set, consider the $\mathrm{NN}$ for the robot (25), (26). The input vector $x_{2}(t)$ can be bounded as

$$
\left\|x_{2}\right\| \leq c_{0}+c_{1}\|z\| .
$$

where $c_{0}, c_{1} \in \mathbb{R}$ are computable positive constants. Let the NN approximation property hold for $f_{2}\left(x_{2}\right)$ given an accuracy $\varepsilon\left(\left\|\varepsilon_{r}\right\| \leq \varepsilon\right)$, for all $x_{2}(t)$ inside a compact set $\Omega_{x_{2}} \triangleq\{x$ : $\left.\|x\| \leq \delta_{r}\right\}$ for $\delta_{r}>c_{0}$, where the size of the set $\Omega_{x_{2}}$ can be increased by increasing the number of hidden layer neurons. Using (39), another compact set can be defined as $\Omega_{c} \triangleq\{x$ : $\left.\|x\| \leq \delta_{r}-c_{0} / c_{1}\right\}$. To ensure that the $\mathrm{NN}$ input $x_{2}(t)$ always 
stays inside the compact set $\Omega_{x_{2}}$, it is sufficient to show that the system states $z(t) \in \Omega_{c} \forall t \geq 0$. First, the initial conditions are chosen to be such that $z(0) \in \Omega_{c}$, which ensures that the NN approximation property holds at time $t=0$. Now using the above Lyapunov stability analysis, it can be shown that $\dot{V}(t)$ is negative whenever $z(t)$ lies outside the compact set $\Omega_{z}$. To prove that $z(t) \in \Omega_{c}$ it is sufficient to show that $\Omega_{z}$ lies completely inside $\Omega_{c}$, which leads to the following gain condition:

$$
\frac{c_{m 1}^{2}}{4 k_{m 1}}+\frac{c_{r 1}^{2}}{4 k_{r 1}}<\beta\left(\frac{\delta_{r}-c_{0}}{c_{1}}\right)^{2}
$$

which can be satisfied by increasing the control gains $k_{m}$ and $k_{r}$. Hence the NN approximation holds throughout provided (40) is satisfied. Similar analysis can be done with the NN used for approximating the environment dynamics in (17).

\section{EXPERIMENTAL RESULTS}

\section{A. Testbed}

The increasing applications involving physical human-robot interaction motivate the development in this brief. For example, in robot gait rehabilitation, a robot may be required to quickly push on a person's limb leading to an impact between the viscoelastic limb and the robot. Towards such an eventual goal, the developed controller was experimentally tested using a two-link robot interacting with a human tissue phantom, as depicted in Fig. 1. The goal of this laboratory-scale bench-top experiment is to examine the performance of the developed controller in a controlled setting with the viscoelastic tissue phantom. In the same spirit as in [7], the DOF of the test apparatus is restricted to two so that additional kinematic complexity is avoided without loss of generality.

The testbed consists of a two-link planar revolute robot (link lengths $l_{1}=0.37 \mathrm{~m}$ and $l_{2}=0.21 \mathrm{~m}$ ) and a mass-spring system (mass $m=30 \mathrm{~kg}$, spring stiffness $=15.1 \mathrm{~N} / \mathrm{m}$ ) that houses the tissue phantom. The body of the mass-spring system includes a U-shaped aluminum plate (item (9) in Fig. 1) mounted on an undercarriage (item (2) in Fig. 1) with porous carbon air bearings (item (3) in Fig. 1) which enables the undercarriage to glide on an air cushion over a glass covered aluminum rail (item (6) in Fig. 1). A steel core spring (item (10) in Fig. 1) connects the U-shaped aluminum plate to an aluminum frame, and a linear variable displacement transducer (LVDT) (item (1) in Fig. 1), held between the aluminum frame and aluminum plate, measures the position of the mass. The HR 2000 series Schaevitz sensor has a nominal linear range of $\pm 50.8 \mathrm{~mm}$ and frequency range from $400 \mathrm{~Hz}$ to $5 \mathrm{kHz}$. The viscoelastic tissue phantom [item (2) in Fig. 1 (Right)], which acts as the impact surface, is held inside a tissue housing [item (8) in Fig. 1 and item (1) in Fig. 1 (Right)], which is connected to the aluminum plate through the sensor housing (item (4) in Fig. 1). The tissue phantom obtained from Simulab Corporation (model TSM-10), models the mechanical behavior of skin and subcutaneous fat. The sensor housing holds a non-contact linear proximity measuring inductive sensor (51U, KD 2440) (item (3) in Fig. 1), from Kaman Measuring Systems, which measures the tissue deformation as the robot impacts the tissue surface. Signal conditioning electronics sense impedance variation as the gap be- tween the sensor and the robot, an electrically conductive target, changes resulting in a displacement signal. In lieu of the inductive sensor used in this experiment, future robotic systems could perhaps use one of a variety of ultrasonic transducers that can be used to measure tissue deformation [30]. The two-link planar robot (items 5 and 7 in Fig. 1) is made of two aluminum links, mounted on $240.0 \mathrm{~N} \cdot \mathrm{m}$ (base link) and $20.0 \mathrm{~N} \cdot \mathrm{m}$ (second link) direct-drive switched reluctance motors. The motors are controlled through power electronics operating in torque control mode. The motor resolvers provide rotor position measurements with a resolution of 614400 pulses/revolution, and a standard backwards difference algorithm is used to numerically determine angular velocity from the encoder readings. A Pentium 2.8 GHz PC operating under QNX hosts the control algorithm. Data acquisition and control implementation were performed at a frequency of $1.0 \mathrm{kHz}$ using the ServoToGo I/O board.

\section{B. Results}

The initial conditions for the robot and the mass position were (in millimeters) $\left(x_{r 1}(0), x_{r 2}(0), x_{m}(0)\right)=$ $(15.4,512.2,164.6)$. The coordinates $x_{r 1}$ and $x_{m}$ lie along the $X_{1}$-axis (the axis along which the mass moves), and $x_{r 2}$ lies along the $X_{2}$-axis of the coordinate system fixed to the ground with origin at the point where the first link of the robot is attached to the base motor. The robot and mass-spring were initially at rest. The desired position of the viscoelastic mass was chosen as $x_{m d}=194.6 \mathrm{~mm}$. The end-effector of the robot was initially $149.2 \mathrm{~mm}$ from the initial position of the viscoelastic mass and $179.2 \mathrm{~mm}$ from the desired setpoint of the mass, along the $X_{1}$-axis. Once the initial impact occurs, the robot is required to move the viscoelastic mass $30 \mathrm{~mm}$ along the $X_{1}$-axis. The control gains were tuned based on user experience as $k_{m}=100, k_{r}=\operatorname{diag}\{257,23\}, \gamma=0.05$, $\alpha=\operatorname{diag}\{12,14\}$. Based on the results of the stability analysis, $k_{m}$ and $k_{r}$ were chosen sufficiently large to decrease the residual error. The gains for the $\mathrm{NN}$ weight adaptation were selected as $\Gamma_{w 1}=8.8 I_{N_{m 2}+1}, \Gamma_{v 1}=16 I_{3}, \Gamma_{w 2}=63 I_{N_{r 2}+1}$, $\Gamma_{v 2}=I_{13}$, where $I_{p} \in \mathbb{R}^{p \times p}$ denotes an identity matrix. The number of hidden layer neurons for the mass-spring and robot system are chosen as $N_{m 2}=5$ and $N_{r 2}=8$, respectively, a choice which reflects a trade-off between performance and computational expense. The initial NN input layer weights $\hat{V}_{1}(0)$ and $\hat{V}_{2}(0)$ are selected randomly such that the activation functions $\sigma_{1}(\cdot)$ and $\sigma_{2}(\cdot)$ form a basis. The output layer weights $\hat{W}_{1}(0)$ and $\hat{W}_{2}(0)$ are both initialized to zero.

Starting from the initial conditions, the robot impacts the viscoelastic mass in approximately $0.9 \mathrm{~s}$. The errors in position for the viscoelastic mass-spring system and the robot are shown in Fig. 2. The peak steady-state position error of the end-effector of the robot along the $X_{1}$-axis $\left|e_{r 1}(t)\right|$ and along the $X_{2}$-axis $\left|e_{r 2}(t)\right|$ are 1.14 and $4.51 \mu \mathrm{m}$, respectively. The peak steady-state position error of the viscoelastic mass $\left|e_{m}(t)\right|$ is $53.8 \mu \mathrm{m}$. The desired robot velocity, which is designed as a virtual control input to the robot is determined from (17). The desired robot trajectory along the $X_{1}$-axis $x_{r d 1}(t)$ is computed by integrating the desired robot velocity $\left(\dot{x}_{r d 1}(t)\right)$, and the desired trajectory along the $X_{2}$-axis is chosen as $x_{r d 2}=361.1$ 
(a)

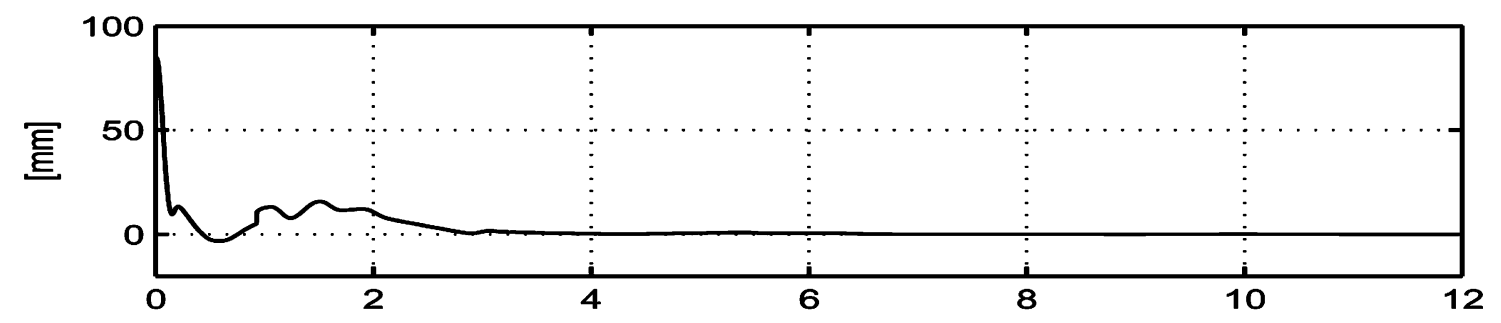

(b)

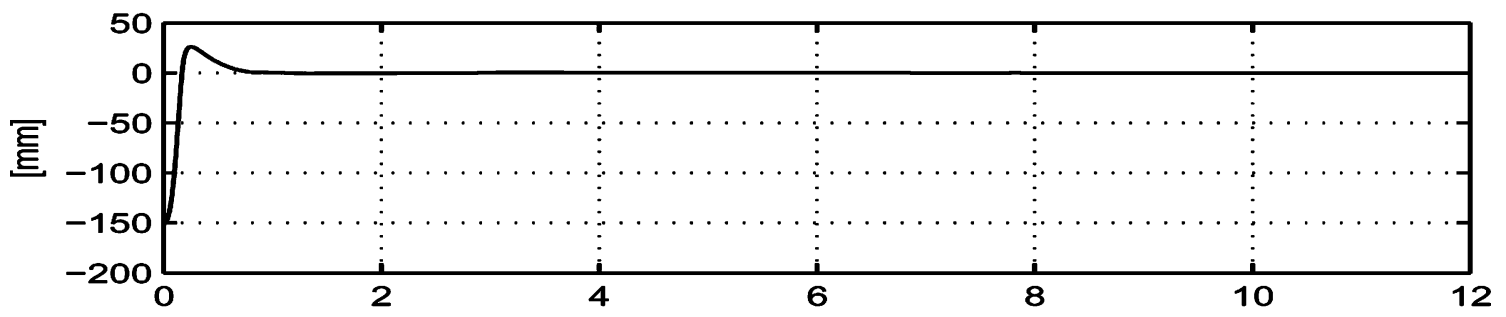

(c)

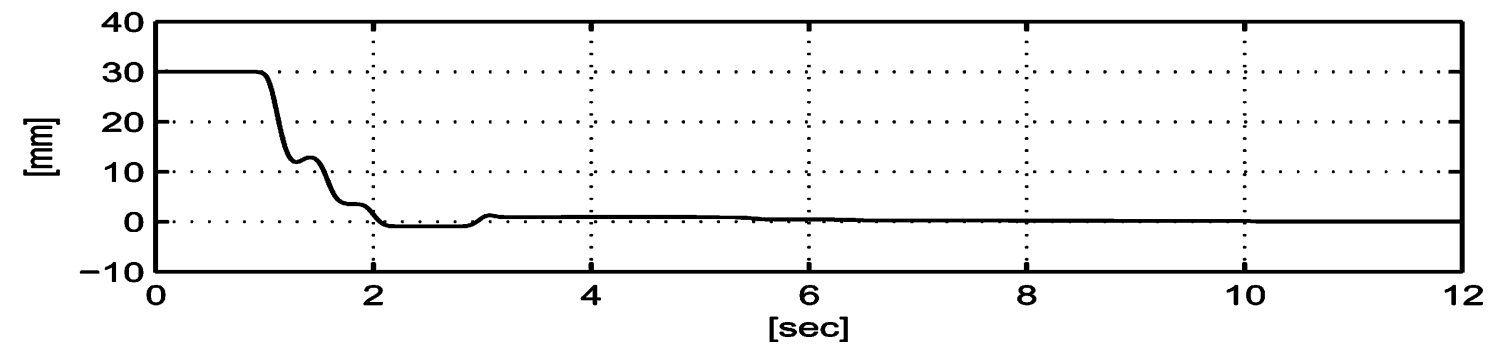

Fig. 2. Mass-spring and robot errors $e(t)$ with the proposed controller. Plot (a) indicates the position error of the robot tip along the $X_{1}$-axis (i.e., $\left.e_{r 1}(t)\right)$, (b) is the position error of the robot tip along the $X_{2}$-axis (i.e., $\left.e_{r 2}(t)\right)$, and (c) is the position error of the mass-spring (i.e., $e_{m}(t)$ ).

$\mathrm{mm}$. The deformation of the tissue phantom $\delta(t)$ during impact is found to be $15 \mathrm{~mm}$ at steady state. Results are compared with a standard PID controller used to regulate the position of the end-effector without any knowledge about the viscoelastic environment. The PID controller has the same initial and final conditions as the proposed controller. Unlike the proposed controller where the desired trajectory for the robot is designed based on approximation of the environment dynamics, the desired set-point of the end-effector with the PID controller is the same as the final mass position. Two PID controllers (PID I and PID II) are implemented. With PID I, the control torques are saturated and the saturation limit kept below the peak torque produced by the proposed controller. The control gains for PID I are chosen as $K_{p}=\operatorname{diag}\{7000,400\} ; K_{i}=\operatorname{diag}\{300,50\}$, $K_{d}=\operatorname{diag}\{200,30\}$, where $K_{p}, K_{i}$ and $K_{d}$ refer to the proportional, integral and derivative components of the PID, respectively. With PID II, the control torques are allowed to be as high as the proposed controller but to ensure safe operation the control gains are chosen lower than PID I, as $K_{p}=$ $\operatorname{diag}\{3500,350\} ; K_{i}=\operatorname{diag}\{300,50\}, K_{d}=\operatorname{diag}\{200,20\}$. A summary of experimental results is provided in Table I.

\section{Discussion}

The experimental results show that the proposed controller ensures stable contact transition while achieving the control objective of regulating the environment to a desired position. Comparison with a PID controllers (I and II) in Table I shows that the
TABLE I

SUMMARIZED EXPERIMENTAL RESULTS

\begin{tabular}{|c|c|c|c|}
\hline Controller & Proposed & PID I & PID II \\
\hline Time to impact $(\mathrm{sec})$ & 0.9 & 0.4 & 0.3 \\
\hline Settling time after impact $(\mathrm{sec})$ & 2.2 & 2.0 & 1.0 \\
\hline Steady state error $e_{m}(\mathrm{~mm})$ & 0.05 & 16.7 & 17.9 \\
\hline RMS error $e_{m}(\mathrm{~mm})$ & 9.5 & 17.6 & 18.3 \\
\hline Robot velocity at impact $(\mathrm{mm} / \mathrm{sec})$ & 81.9 & 318.4 & 319.5 \\
\hline
\end{tabular}

PID has a higher velocity at impact, more steady-state error, and a better transient response than the proposed controller. These results can be attributed to the fact that the PID uses no knowledge of the environment and hence cannot account for the viscoelastic effects.

The experimental results provide insight into practical implementation issues with the proposed controller. The use of the inductive sensor to measure the tissue deformation is not likely feasible in a practical scenario. However, as mentioned in the Section V-A, the use of a non-contact ultrasonic sensor, commonly used for studying tissue characteristics, can be used in lieu of the inductive sensor. The development assumes in this work that the contact is perfectly detected by the inductive sensor which may not be possible in every scenario because of issues related to sensor placement and sensor noise. It would be interesting to explore some robust mechanisms of detecting impact wherein the controller is robust to inaccuracies in detecting impact. An alternative approach could be to use a vision-based 
(a)

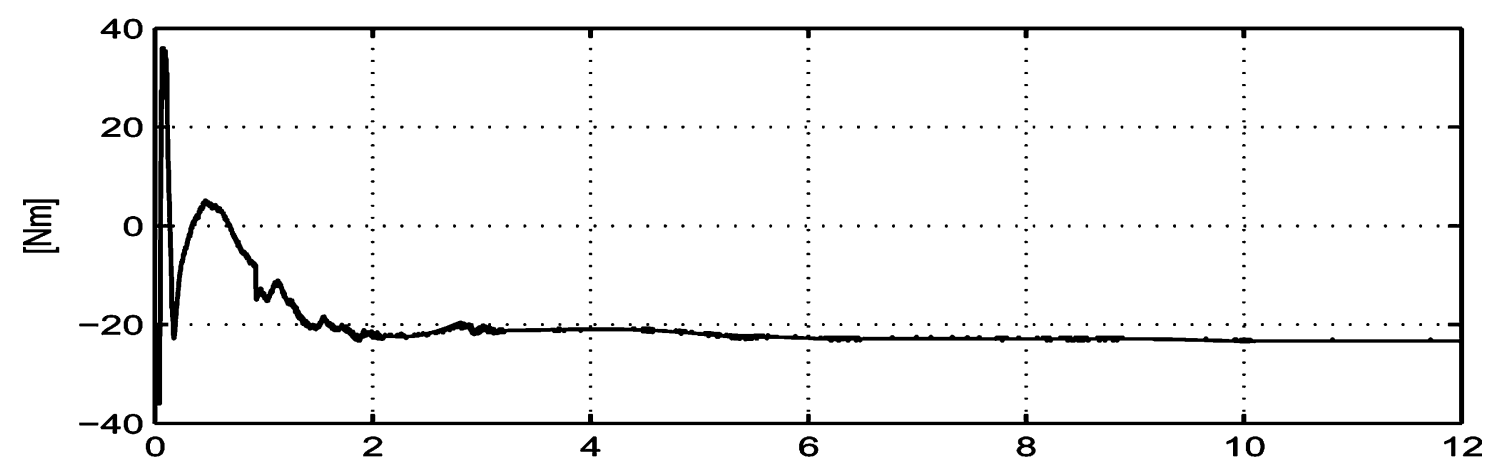

(b)

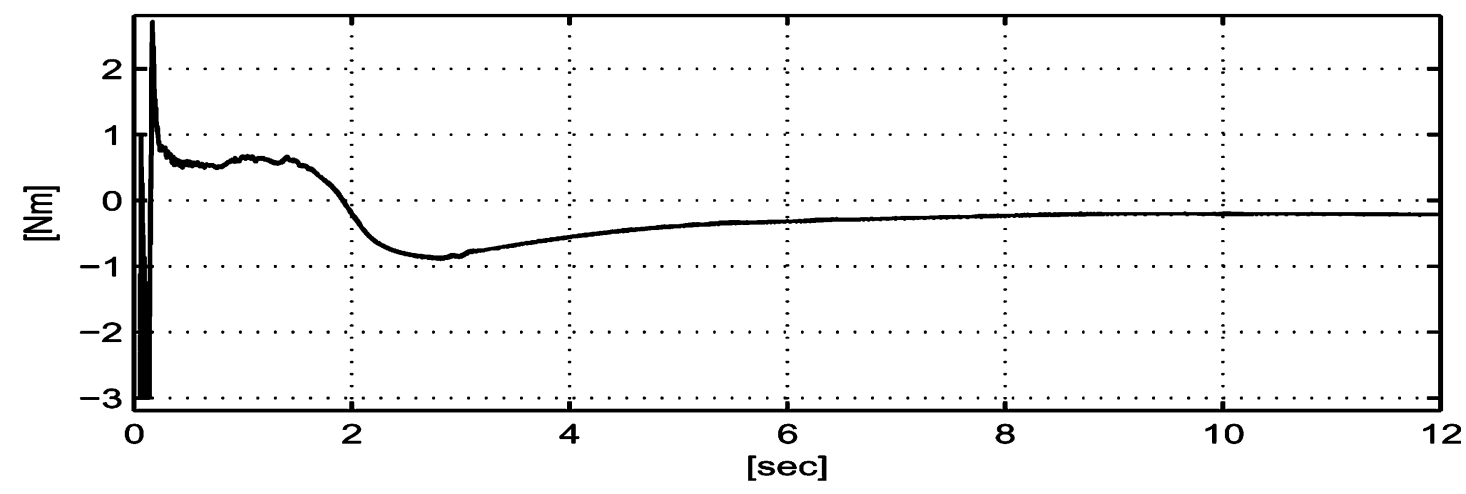

Fig. 3. Applied control torques $J^{T}(q) F(t)$, with the proposed controller, for the (a) base motor and (b) second link motor.

method for detecting impact. Although the size of the UUB region can be made arbitrarily small by increasing the gains $k_{m}$ and $k_{r}$, high gain feedback can result in a higher impact force which is undesirable for human-robot interaction. Our future work [31] attempts to overcome this problem of limiting the impact force by saturating the feedback terms of the controller. A question also arises when choosing the number of hidden layer neurons. This choice involves a tradeoff between the approximation accuracy of the NN and the computational expense of increasing the number of neurons. In the experiment, the number of neuron was limited to $N_{m 2}=5$ and $N_{r 2}=8$ for successful real-time operation of the control algorithm at $1 \mathrm{KHz}$ for the aforementioned computational platform. It was observed that increasing the NN gains made the neural network learn faster; however, increasing the gains beyond a certain limit caused the mass to overshoot the desired position. Slight improvement in the transient response was further achieved by increasing the number of hidden layer neurons of the NN, but this led to an increased computational burden. A hybrid control strategy (e.g., [19]), may be an alternative for improving the transient performance.

\section{CONCLUSION}

Unlike previous contact transition problems, the aim of this research is to control the states of an unactuated dynamic system through an impact collision by another dynamic system (robot). A possible use of robots for human-robot interaction motivates the development in this brief. The use of NNs makes it possible to include an uncertain non-LP physically consistent contact model (Hunt-Crossley) in the error system dynamics. A
Lyapunov-based backstepping approach is used to generate the desired robot velocity signal and a control input to the robot is designed to ensure stability. Experimental results of a robot interacting with a tissue phantom show that the controller ensures a stable interaction with the viscoelastic environment. Practical implementation issues are also discussed in this brief.

\section{REFERENCES}

[1] H. Hertz, Hertzs Miscellaneous Papers-Chapters 5 and 6. London, U.K.: MacMillan, 1896.

[2] S. Dubowsky and F. Freudenstein, "Dynamic analysis of mechanical systems with clearances-part 1: Formation of dynamical model," $J$. Eng. for Ind., vol. 93, pp. 305-309, 1971.

[3] K. Hunt and F. Crossley, "Coefficient of restitution interpreted as damping in vibroimpact," ASME J. Appl. Mechan., vol. 42, no. 2, pp. 440-445, 1975.

[4] D. W. Marhefka and D. E. Orin, "A compliant contact model with nonlinear damping for simulation of robotic systems," IEEE Trans. Syst., Man, Cybern., A, Cybern., vol. 29, no. 6, pp. 566-572, Nov. 1999.

[5] N. Diolaiti, C. Melchiorri, and S. Stramigioli, "Contact impedance estimation for robotic systems," IEEE Trans. Robot. Autom., vol. 21, no. 5, pp. 925-935, Oct. 2005.

[6] G. Gilardi and I. Sharf, "Literature survey of contact dynamics modelling," Mechan. Mach. Theory, vol. 37, no. 10, pp. 1213-1239, 2002.

[7] N. Hogan, "Stable execution of contact tasks using impedance control," in Proc. IEEE Conf. Robot. Autom., Mar. 1987, vol. 4, pp. 1047-1054.

[8] M. Raibert and J. Craig, "Hybrid position/force control of manipulators," J. Dyn. Syst., Meas., Control, vol. 102, no. 127, pp. 126-133, 1981.

[9] K. Youcef-Toumi and D. Gutz, "Impact and force control: Modeling and experiments," J. Dyn. Syst., Meas., Control, vol. 116, pp. 89-89, 1994.

[10] J. K. Mills and D. M. Lokhorst, "Stability and control of robotic manipulators during contact/noncontact task transition," IEEE Trans. Robot. Autom., vol. 9, no. 3, pp. 335-345, Jun. 1993.

[11] T.-J. Tarn, Y. Wu, N. Xi, and A. Isidori, "Force regulation and contact transition control," IEEE Control Syst. Mag., vol. 16, no. 1, pp. 32-40, Feb. 1996. 
[12] P. R. Pagilla and B. Yu, "A stable transition controller for constrained robots," IEEE/ASME Trans. Mechatron., vol. 6, no. 1, pp. 65-74, Mar. 2001.

[13] P. Akella, V. Parra-Vega, S. Arimoto, and K. Tanie, "Discontinuous model-based adaptive control for robots executing free and constrained tasks," in Proc. IEEE Conf. Robot. Autom., May 1994, pp. 3000-3007.

[14] A. Tornambe, "Modeling and control of impact in mechanical systems: Theory and experimental results," IEEE Trans. Autom. Control, vol. 44, no. 2, pp. 294-309, Feb. 1999.

[15] R. Volpe and P. Khosla, "A theoretical and experimental investigation of impact control for manipulators," Int. J. Robot. Res., vol. 12, no. 4, pp. 351-351, 1993.

[16] K. Dupree, C. Liang, G. Hu, and W. Dixon, "Adaptive lyapunov-based control of a robot and mass-spring system undergoing an impact collision," IEEE Trans. Syst., Man, Cybern., B, Cybern., vol. 38, no. 4, pp. 1050-1061, 2008.

[17] S. Bhasin, K. Dupree, Z. D. Wilcox, and W. E. Dixon, "Adaptive control of a robotic system undergoing a non-contact to contact transition with a viscoelastic environment," in Proc. Amer. Controls Conf., 2009, pp. 3506-3511.

[18] S. Bhasin, K. Dupree, P. M. Patre, and W. E. Dixon, "Neural network control of a robot interacting with an uncertain Hunt-Crossley viscoelastic environment," in Proc. ASME Dyn. Syst. Control Conf., 2008, pp. 875-882.

[19] R. Carloni, R. Sanfelice, A. Teel, and C. Melchiorri, "A hybrid control strategy for robust contact detection and force regulation," in Proc. Amer. Control Conf., 2007, pp. 1461-1466.

[20] Z. Doulgeri and G. Iliadis, "Stability of a contact task for a robotic arm modelled as a switched system," Control Theory Appl. IET, vol. 1, no. 3, pp. 844-853, 2007.

[21] Z. Li and W. Chen, "Adaptive neural-fuzzy control of uncertain constrained multiple coordinated nonholonomic mobile manipulators," Eng. Appl. Artif. Intell., vol. 21, no. 7, pp. 985-1000, 2008.
[22] S. Bhasin, K. Dupree, and W. E. Dixon, "Control of Robotic Systems Undergoing a Non-Contact to Contact Transition," in , Robot Manipulators, M. Ceccarelli, Ed. Vienna, Austria: In-Tech, 2008, ch. 6 [Online]. Available: http://intechweb.org/book.php?id=83

[23] C. Liang, S. Bhasin, K. Dupree, and W. E. Dixon, "A force limiting adaptive controller for a robotic system undergoing a non-contact to contact transition," IEEE Trans. Control Syst. Technol., vol. 17, no. 6 , pp. 1330-1341, Nov. 2009.

[24] F. Salah and W. Holly, "Modeling of impact dynamics: A literature survey," in Proc. Int. ADAMS User Conf., 2000, vol. 80.

[25] F. Lewis, D. Dawson, and C. Abdallah, Robot Manipulator Control: Theory and Practice. Boca Raton, FL: CRC Press, 2004.

[26] F. Lewis, J. Campos, and R. Selmic, Neuro-Fuzzy Control of Industrial Systems with Actuator Nonlinearities. Warrendale, PA: Society for Industrial Mathematics, 2002.

[27] M. Krstic, P. Kokotovic, and I. Kanellakopoulos, Nonlinear and Adaptive Control Design. New York: Wiley, 1995.

[28] P. Patre, W. MacKunis, K. Kaiser, and W. Dixon, "Asymptotic tracking for uncertain dynamic systems via a multilayer neural network feedforward and rise feedback control structure," IEEE Trans. Autom. Control, vol. 53, no. 9, pp. 2180-2185, Sep. 2008.

[29] H. Khalil, Nonlinear Systems. Upper Saddle River, NJ: Prentice-Hall, 2002.

[30] L. Han, J. Noble, and M. Burcher, "A novel ultrasound indentation system for measuring biomechanical properties of in vivo soft tissue," Ultrasound in Med. Biol., vol. 29, no. 6, pp. 813-823, 2003.

[31] S. Bhasin, P. M. Patre, Z. Kan, and W. E. Dixon, "Control of a robot interacting with an uncertain viscoelastic environment with adjustable force bounds," in Proc. Amer. Control Conf., 2010, pp. 5242-5247. 